\title{
VARIABILIDADE DA FREQUÊNCIA CARDÍACA COMO MARCADOR FUNCIONAL DO DESENVOLVIMENTO
}

\author{
HEART RATE VARIABILITY AS A FUNCTIONAL MARKER OF \\ DEVELOPMENT
}

\author{
Luiz Carlos de Abreu*
}

O crescimento normal inclui a maturação e o aumento do tamanho do corpo. O ritmo e a velocidade dessas mudanças variam de pessoa para pessoa e são condicionados por fatores hereditários e ambientais ${ }^{1}$. A maturidade física inicia-se, na atualidade, mais cedo do que há um século, provavelmente devido à melhoria da nutrição, da saúde em geral e das condições de vida.

Já o desenvolvimento é a capacidade progressiva do ser humano em realizar funções cada vez mais complexas ${ }^{2}$. Este processo é o resultado da interação entre os fatores biológicos, próprios da espécie e do indivíduo e os fatores culturais, próprios do meio social onde esse indivíduo encontrase inserido.

A maturação do sistema nervoso central e consequente integridade dos reflexos, o desempenho nos aspectos cognitivos, na inteligência, adaptação, inter-relação com o meio ambiente e constituição do psiquismo são aspectos que confirmam que o desenvolvimento vaialém de uma determinação biológica e necessita uma abordagem multiconceitual e, consequentemente, multidisciplinar $^{3}$

Assim, a aquisição de novas habilidades está diretamente relacionada não apenas à faixa etária da criança, mas também às interações vividas com os outros seres humanos do seu grupo social, caracterizando o desenvolvimento como fator de relações bidirecionais em que a criança influencia e é influenciada por aqueles que a circundam.

O sistema nervoso autônomo (SNA) desempenha papel fundamental no crescimento e desenvolvimento, provendo condições para que os indivíduos estejam preparados para superar as dificuldades de adaptação inerentes à idade. Nesta linha, o SNA corrobora para importante papel na regulação dos processos fisiológicos do organismo humano tanto em condições normais quanto patológicas ${ }^{4}$.

Faz-se, assim, necessário o desenvolvimento de técnica simples e confiável para melhor avaliar esses processos do desenvolvimento a partir do retrato de variável biológica ${ }^{4}$. Dentre as técnicas utilizadas para sua avaliação, a variabilidade da frequência cardíaca (VFC) tem emergido como uma medida simples e não invasiva dos impulsos autonômicos, representando um dos mais promissores marcadores quantitativos do balanço autonômico. A VFC descreve as oscilações no intervalo entre batimentos cardíacos consecutivos (intervalos RR), assim como oscilações entre frequências cardíacas instantâneas consecutivas ${ }^{4.5}$, estando portanto na dependência da atividade do sistema nervoso simpático e parassimpático ${ }^{6,7}$. Esta pode ser avaliada tanto por meio de analises lineares, no domínio do tempo e no domínio da frequência, quanto por meio de análises não lineares baseadas no domínio do caos $^{4,5}$.

Trata-se de uma medida que pode ser utilizada para avaliar a modulação do SNA sob condições fisiológicas, tais como em situações de vigília e sono, diferentes posições do corpo, treinamento físico, e também em condições patológicas. Uma alta variabilidade na frequência cardíaca é sinal de boa adaptação, caracterizando um indivíduo saudável, com mecanismos autonômicos eficientes, enquanto que baixa variabilidade é frequentemente um indicador de adaptação anormal e insuficiente do SNA,

\footnotetext{
Laboratório de Delineamento Estudos e Escrita Científica da Faculdade de Medicina do ABC. Corresponding author: abreu.luizcarlos@gmail.com

Suggested citation: Abreu LC. Heart rate variability as a functional marker of development. J. Hum. Growth Dev. 2012; 22(3): 279-282

Manuscript submitted Oct 04 2012, accepted for publication Oct 102012.
} 
implicando a presença de mau funcionamento fisiológico no indivíduo.

Diversos estudos analisaram o comportamento do SNA frente à obesidade e utilizaram, para esta análise, a VFC. Várias pesquisas têm abordado esta questão, entre as quais fazendo associação com variáveis fisiológicas e farmacológicas ${ }^{8}$.

O potencial de utilização de VFC induzido por um estímulo auditivo musical, como um indicador clínico para avaliar e identificar problemas de saúde que envolve mudanças autonômicas, é promissor ${ }^{9}$. Esta técnica pode ser utilizada como um instrumento para o diagnóstico precoce e prognóstico da disfunção autonômica em indivíduos expostos a sons intensos durante longos períodos ${ }^{4-11}$.

No estudo de Alves et al. ${ }^{5}$ houve redução da atividade parassimpática na avaliação da VFC durante testes autonômicos cardiovasculares e a capacidade funcional de indivíduos com Diabetes Mellitus tipos 1 e $2^{5}$. Já em crianças obesas houve modificações no SNA, que, de acordo com os autores $^{7}$, demonstram a necessidade de atenção precoce a esta população para evitar complicações futuras no crescimento e desenvolvimento ${ }^{7}$.

Ademais, o aumento da incidência e prevalência de crianças com sobrepeso/obesidade tem sido um motivo de preocupação nos últimos anos, devido ao crescente corpo de conhecimento que ligam á alimentação e estilos de vida sedentários a aumento de peso e de gordura corporal em crianças ${ }^{11}$ e, além disso, implicações de curto e longo prazo da saúde, incluindo alterações na função autonômica cardíaca ${ }^{12}$. Há sugestão de reduzida VFC em crianças com sobrepeso/obesidade ${ }^{13}$.
Variabilidade da frequência cardíaca é instrumento importante para avaliar a saúde do coração. Redução da VFC está associada com aumento da morbidade e mortalidade de doenças cardiovasculares ${ }^{14}$, doença cardíaca congênita ${ }^{15}$, neuropatia diabética ${ }^{16} \mathrm{e}$ outras doenças e processos fisiológicos. Há falta geral de literatura existente sobrea VFC em crianças aparentemente saudáveis, especialmente em relação ao seu status de peso. Compreender como a VFC está associada com excesso de peso/ obesidade pode ser essencial no planejamento das intervenções dirigidas a esforços de prevenção para melhorar a saúde cardiovascular e reduzir excesso de peso e obesidade em crianças.

Mudanças nos padrões da VFC fornecem um indicador sensível e antecipado de comprometimentos na saúde4. Alta VFC é sinal de boa adaptação, caracterizando um indivíduo saudável com mecanismos autonômicos eficientes4. Inversamente, baixa VFC é frequentemente um indicador de adaptação anormal e insuficiente do SNA, o que pode indicar a presença de mau funcionamento fisiológico no indivíduo, necessitando de investigações adicionais de modo a encontrar um diagnóstico específico ${ }^{10}$.

A VFC é um importante instrumento de avaliação do SNA, o qual tem um importante papel na manutenção da homeostase. Sua utilização é diversificada e se firma como preditor das funções internas do organismo, tanto em condições normais quanto patológicas, caracterizando-se como instrumento atual de avaliação e identificação dos comprometimentos na saúde, no crescimento e desenvolvimento humano. Eis uma linha de pesquisa importante e atual, com foco na saúde da criança e do adolescente. 


\section{REFERÊNCIAS:}

1. WHO, Physical Status: the use and interpretation of anthropometry. Geneva: Report of a WHO Expert Committee, 1995. (WHO Technical Report Series, 854).

2. MARCONDES, E. Pediatria básica. $8^{a}$ ed. São Paulo: Sarvier, 1994.

3. Brasil. Ministério da Saúde. Secretaria de Políticas de Saúde. Departamento de Atenção Básica. Saúde da criança: acompanhamento do crescimento e desenvolvimento infantil / Ministério da Saúde. Secretaria de Políticas de Saúde. Brasília: Ministério da Saúde, 2002.

4. Vanderlei LCM, Pastre CM, Hoshi RA, Carvalho TD, Godoy MF. Noções básicas de variabilidade da frequência cardíaca e sua aplicabilidade clínica. Rev Bras Cir Cardiovasc 2009; 24(2): 205-17.

5. Alves, RL et al. Autonomic Modulation and Functional Capacity in Diabetes Mellitus Type 1 and 2 Subjects. J. Hum. Growth Dev. 2012; 22(1): 327-333

6. Longo A, Ferreira D, Correia MJ. Variabilidade da Freqüência Cardíaca. Rev. PortCardiol. 1995;14(3):241-262.

7. Souza NM, et al. Heart rate variability in obese children. J. Hum. Growth Dev. 2012; 22(3): 334-339.

8. Task Force of European Society of Cardiology of the North American Society of Pacing Electrophysiology. Heart rate variability. Standars of mensurement, physiological interpretation and clinical use.Circulation. 1996;93:1043-65.
9. Valenti Vitor E., Guida Heraldo L., Frizzo Ana C. F., Cardoso Ana C. V., Vanderlei Luiz Carlos M., Abreu Luiz Carlos de. Auditory stimulation and cardiac autonomic regulation. Clinics, 67(8): 955-958. http://dx.doi.org/10.6061/clinics/ 2012(08)16

10. Pumprla J, Howorka K, Groves D, Chester M, Nolan J. Functional assessment of heart rate variability: physiological basis and practical applications. Int J Cardiol. 2002;84(1):1-14.

11. Lobstein $\mathrm{T}$, Leach R. Foresight. Tackling Obesities: Future Choices - International Comparisons of Obesity Trends, Determinants and Responses - Evidence Review.Government Office for Science, London (2007).

12. Vanderlei LCM, Pastre CM, Godoy M et al. Fractal correlation of heart rate variability in obese children. Auton. Neurosci. Basic Clin., 155 (2010), pp. 125-129.

13. Samantha L. Birch, Michael J. Duncan, Craig Franklin. Overweight and reduced heart rate variability in British children: An exploratory study.Preventive Medicine, Available online 23 September 2012. Available online 23 September 2012. http://dx.doi.org/10.1016/j.ypmed. 2012.09.015

14. Kluttig A, Schumann B, Swenne CA, Kors JA, Kuss et al. Association of health behaviour with heart rate variability: a population-based study. BMC Cardiovasc.Disord., 25 (10) (2010), p. 5

15. N.P. Heragu NP, Scott WA. Heart rate variability in healthy children and in those with congenital heart disease both before and after operation Am. J. Cardiol., 83 (1999), pp. 1654-1657

16. Kautzner J, Camm J. Clinical relevance of heart rate variability. Clin.Cardiol., 20 (1997), pp. 162-168. 
